\title{
Universiteit
}

Leiden

The Netherlands

\section{D nanometer-scale study of coexisting bicontinuous morphologies in a block copolymer/homopolymer blend}

Jinnai, H.; Hasegawa, H.; Nishikawa, Y.; Sevink, G.J.A.; Braunfeld, M.B.; Agard, D.A.; Spontak, R.J.

\section{Citation}

Jinnai, H., Hasegawa, H., Nishikawa, Y., Sevink, G. J. A., Braunfeld, M. B., Agard, D. A., \& Spontak, R. J. (2006). 3D nanometer-scale study of coexisting bicontinuous morphologies in a block copolymer/homopolymer blend. Macromolecular Rapid Communications, 27(17), 1424-1429. doi:10.1002/marc.200600344

Version: $\quad$ Publisher's Version

License: $\quad$ Licensed under Article 25fa Copyright Act/Law (Amendment Taverne)

Downloaded from: https://hdl.handle.net/1887/3193714

Note: To cite this publication please use the final published version (if applicable). 
Summary: Coexisting bicontinuous morphologies, one ordered and one disordered, are investigated in a macrophaseseparated poly(styrene-block-isoprene) diblock copolymer/ homopolystyrene (SI/hS) blend. Two-phase behavior is attributed to the relatively high $\mathrm{hS} / \mathrm{S}$ mass ratio (0.92). According to its crystallographic signature and channel coordination as discerned from three-dimensional (3D) images generated by transmission electron microtomography (TEMT), the ordered morphology is classified as gyroid. The 3D local and global topological characteristics of both bicontinuous morphologies as measured directly from TEMT images are reported. The disordered morphology is further compared with molecularfield simulations to ascertain the spatial distribution of the constituent species within the blend, thereby demonstrating the utility of high-resolution 3D imaging coupled with molecularlevel simulations.
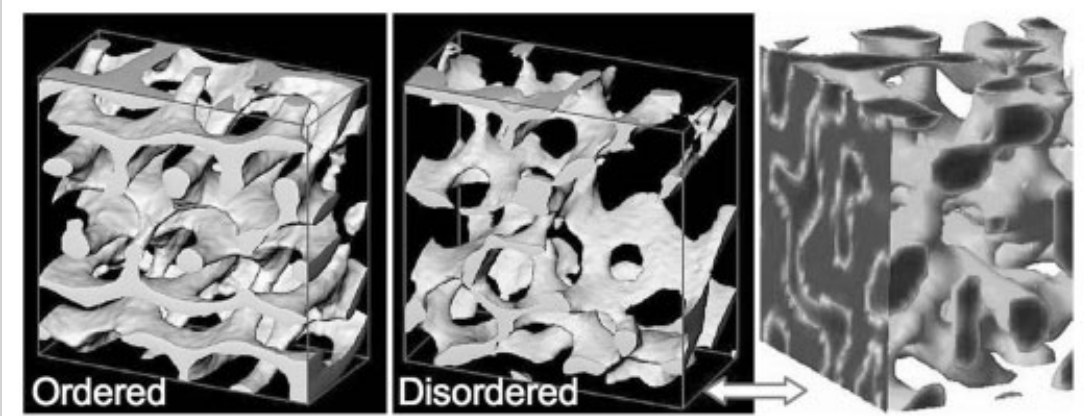

\title{
3D Nanometer-Scale Study of Coexisting Bicontinuous Morphologies in a Block Copolymer/ Homopolymer Blend
}

\author{
Hiroshi Jinnai, ${ }^{1}$ Hirokazu Hasegawa, ${ }^{2}$ Yukihiro Nishikawa, ${ }^{1}$ G. J. Agur Sevink,${ }^{3}$ Michael B. Braunfeld, ${ }^{4}$ \\ David A. Agard, ${ }^{4}$ Richard J. Spontak ${ }^{* 5}$ \\ ${ }^{1}$ Department of Polymer Science \& Engineering, Kyoto Institute of Technology, Kyoto 606-8585, Japan \\ ${ }^{2}$ Department of Polymer Chemistry, Kyoto University, Kyoto 606-8501, Japan \\ E-mail: hasegawa@alloy.polym.kyoto-u.ac.jp \\ ${ }^{3}$ Leiden Institute of Chemistry, Leiden University, 2300 RA Leiden, The Netherlands \\ ${ }^{4}$ Departments of Biochemistry \& Biophysics and the Howard Hughes Medical Institute, University of California, \\ San Francisco, CA 94143, USA \\ ${ }^{5}$ Departments of Chemical \& Biomolecular Engineering and Materials Science \& Engineering, \\ North Carolina State University, Raleigh, NC 27695, USA \\ E-mail: Rich_Spontak@ncsu.edu
}

Received: May 16, 2006; Accepted: June 19, 2006; DOI: 10.1002/marc.200600344

Keywords: bicontinuous networks; block copolymers; phase separation; simulations; TEM

\section{Introduction}

Immiscible homopolymers commonly undergo macrophase separation because of endothermic mixing, in conjunction with a negligible entropy of mixing, at relatively large length scales. Covalent coupling of two such homopolymers yields a diblock copolymer, which can likewise undergo phase segregation, but at much smaller length scales. Because of its physically restricted blocks, an incompatible diblock copolymer can spontaneously self-organize into several ordered nanometer-scale morphologies, which include spheres on a body- or face-centered cubic lattice, cylinders on a hexagonal lattice, bicontinuous channels, and alternating lamellae. ${ }^{[1]}$ Tunable morphological control is crucial to 
emerging nanotechnologies ${ }^{[2,3]}$ and reflects entropic differences associated with interfacial chain packing. ${ }^{[4]}$ The morphologies of microphase-ordered diblock copolymers can be chemically altered by synthesizing ${ }^{[5]}$ copolymer molecules that differ in composition, repeat unit asymmetry, ${ }^{[6]}$ or (liquid) crystallinity. ${ }^{[7,8]}$ Alternatively, interfacial chain packing and, hence, morphology can be physically adjusted through the addition of a second (miscible) species, such as a selective solvent, ${ }^{[9]}$ a parent homopolymer, or a second copolymer. ${ }^{[10]}$ Of particular interest here are the bicontinuous morphologies generated thus far in block copolymer systems.

Although ordered bicontinuous morphologies such as perforated lamellae ${ }^{[11]}$ and the 'Plumber's Nightmare' ${ }^{[12]}$ have been reported, the gyroid $(\mathrm{G})$ motif with $I a \overline{3} d$ symmetry is the most ubiquitous ordered bicontinuous morphology observed in neat linear ${ }^{[13-15]}$ and non-linear ${ }^{[16]}$ block copolymer melts, as well as in blends of copolymers with solvents, ${ }^{[17]}$ homopolymers, ${ }^{[18]}$ or other copolymers. ${ }^{[19]}$ Accurate classification of this complex morphology has relied upon mesoscale crystallography, ${ }^{[13,14]}$ minimal-surface modeling, ${ }^{[14]}$ and transmission electron microtomography (TEMT). ${ }^{[20 \mathrm{a}]}$ In the presence of at least one added parent homopolymer, block copolymer molecules can form other bicontinuous morphologies. Aperiodic bicontinuous morphologies composed of bilayered channels and generically referred to as 'sponge' morphologies have been reported ${ }^{[21,22]}$ for triblock copolymers in the presence of a single homopolymer. In ternary systems composed of two homopolymers with typically less than 10 wt.-\% block copolymer, a microemulsion morphology develops at the Lifshitz point where microphase and macrophase separation are equally favored. ${ }^{[23]}$ Previous studies ${ }^{[24]}$ have likewise reported on the existence of complex network morphologies in block copolymer systems. In general, such bicontinuous and network morphologies appear either ordered or disordered, but not both.

The objective of this work is to examine the bicontinuous morphology that results by adding a relatively high-molecular-weight homopolymer (hA) to a microphase-ordered AB diblock copolymer. Using self-consistent field theory (SCFT), Matsen ${ }^{[25]}$ has generated phase diagrams for AB/ $\mathrm{hA}$ blends that differ in thermodynamic incompatibility $(\chi N$, where $\chi$ is the Flory-Huggins interaction parameter, and $N=N_{\mathrm{A}}+N_{\mathrm{B}}$ is the number of statistical units along the copolymer backbone) and homopolymer/copolymer chain length ratio ( $\left.\alpha=N_{\mathrm{hA}} / N_{\mathrm{AB}}\right)$. The SCFT phase diagram that corresponds to $\chi N=11$ and $\alpha=0.67$ shows that, in addition to the single morphologies described earlier, coexisting morphologies and a two-phase (2Ф) region that extends over most of the homopolymer composition range $\left(\phi_{\mathrm{hA}}\right)$ at low- to mid-copolymer compositions $\left(f_{\mathrm{A}}=N_{\mathrm{A}} / N\right)$ are also predicted to exist. The $2 \Phi$ region is found ${ }^{[25]}$ to enlarge (to higher $f_{\mathrm{A}}$ and lower $\left.\phi_{\mathrm{hA}}\right)$ as both $\alpha$ and $\chi N$ increase. Experimental studies of copolymer-rich $\mathrm{AB} / \mathrm{hA}$ blends performed to date have focused extensively on miscible blends, wherein added hA molecules residing within and wetting the stretched A blocks of the ordered copolymer dictate geometrical and dimensional characteristics. Although prior theoretical and experimental studies provide valuable guidance regarding copolymer/homopolymer blends, not all phase space has yet been investigated, and questions regarding the phase behavior of such blends near expected transitions linger.

\section{Experimental Part}

A poly(styrene-block-isoprene) (SI) diblock copolymer and a polystyrene homopolymer (hS) were synthesized via living anionic polymerization. The molecular weight and composition of the copolymer, measured by gel permeation chromatography (GPC) and ${ }^{1} \mathrm{H}$ NMR spectroscopy, respectively, were $100 \mathrm{kDa}$ and 60 wt.-\% (57 vol.-\%) S, whereas the molecular weight of the hS was $55 \mathrm{kDa}$, so that $\alpha=0.48$ (corrected for $\mathrm{S}$ and I mass densities). This translates to a $\mathrm{hS} / \mathrm{S}$ mass ratio of 0.92 . The polydispersity indices from gel permeation chromatography (GPC) were 1.19 (SI) and $1.04(\mathrm{hS})$. A binary 68/32 w/w SI/hS blend, which resides near the transition from ordered morphologies to disordered micelles in a previously reported ${ }^{[18]}$ block copolymer/homopolymer phase diagram, was prepared by dissolving both species at $5 \% \mathrm{w} / \mathrm{v}$ in toluene. Upon solution casting, the solvent was slowly removed over the course of two weeks, and the resultant film was further dried under vacuum for $2 \mathrm{~d}$ at ambient temperature and subsequently microtomed at $-100{ }^{\circ} \mathrm{C}$. Sections measuring $\approx 200 \mathrm{~nm}$ thick were stained with $2 \% \mathrm{OsO}_{4}(\mathrm{aq})$ vapor for $30 \mathrm{~min}$, decorated with $15 \mathrm{~nm} \mathrm{Au}$ nanoparticles, and then coated with carbon. Transmission electron microscopy (TEM) images were collected at tilt $(\theta)$ angles that ranged from $-65^{\circ}$ to $+65^{\circ}$ at an angular interval of $1^{\circ}$ on a Technai T20 microscope operated at $200 \mathrm{kV}$. Images were acquired at a resolution of $0.89 \mathrm{~nm} \cdot \operatorname{pixel}^{-1}$ on a Gatan UltraScan 4000 CCD camera and aligned using the Au fiducial markers. Volumetric TEMT reconstructions were generated $^{[26]}$ from the filtered back-projection algorithm in the EMCAT software suite.

\section{Results and Discussion}

ATEM image collected from the blend at $0^{\circ}$ tilt is presented in Figure 1 and reveals that the blend exhibits two distinct bicontinuous morphologies, one ordered and the other disordered. The ordered one resembles the $\mathrm{G}$ morphology viewed along the (111) projection axis. Although $\mathrm{G}$ can coexist with other non-bicontinuous morphologies such as cylinders, we are unaware of any reports wherein ordered and disordered bicontinuous phases coexist. Direct comparison with prior SCFT predictions is not possible because of differences in both $\chi N$ (estimated as $\approx 77$ from the temperature-dependent $\chi$ reported by Lodge et al. ${ }^{[27]}$ and density-corrected monodisperse block lengths) and, to a lesser extent, $\alpha$. Attempts to perform SCFT calculations for the present blend conditions of $\chi N$ and $\alpha$ have been 


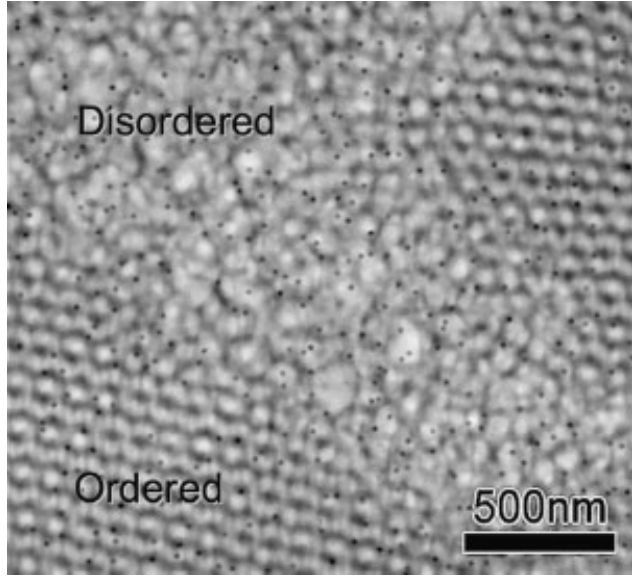

Figure 1. ATEM image of the blend under current investigation (with $\alpha=0.48$ ), which confirms the presence of two coexisting bicontinuous morphologies, one ordered and the other disordered. In this and subsequent images, the I microdomains appear dark because of selective $\mathrm{OsO}_{4}$ staining. The $15 \mathrm{~nm}$ Au nanoparticles used for image alignment purposes are visible in this image.

unsuccessful because of stability problems. Another factor to be considered (beyond the scope of this work) is copolymer polydispersity, since Sides and Fredrickson ${ }^{[28]}$ have shown that block copolymer morphologies can be affected by variations in molecular weight distribution. The present copolymer/homopolymer blend nonetheless provides a unique opportunity to investigate bicontinuous morphologies that differ in spatial organization. Detailed analysis of these coexisting phases can help to elucidate key morphological differences and similarities, as well as bridge contemporary studies of ordered and disordered bicontinuous morphologies.

Results from the TEMT analysis are displayed in Figure 2. Two-dimensional planar and cross-sectional slices of the three-dimensional (3D) volume elements generated by reconstructing independent series of tilt images collected from the ordered and disordered morphologies are provided for comparison in Figure 2a and 2b, respectively, whereas corresponding solid renderings are included in Figure 2c and $2 \mathrm{~d}$. For reference, the $z$ direction in these montages is orthogonal to the specimen surface along $x$ and $y$. A marked difference in nanostructural order between the two morphologies is evident from the image slices in Figure $2 \mathrm{a}$ and $2 \mathrm{~b}$. Close examination of the solid renderings displayed in Figure $2 \mathrm{c}$ and $2 \mathrm{~d}$ further reveals that, while the ordered morphology consists primarily of channels that appear circular in cross-section, some of the I microdomains in the disordered morphology appear more layer-like. Dimensional changes incurred as a consequence of beam damage during a)

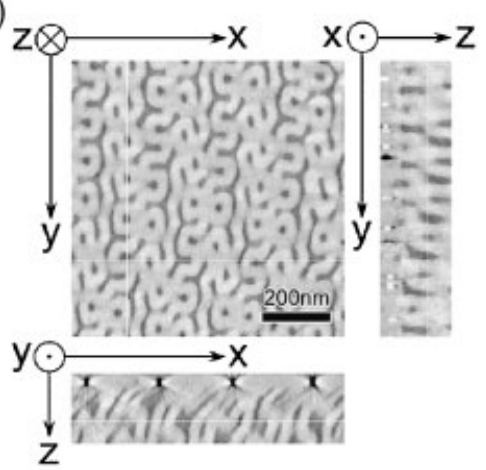

b)

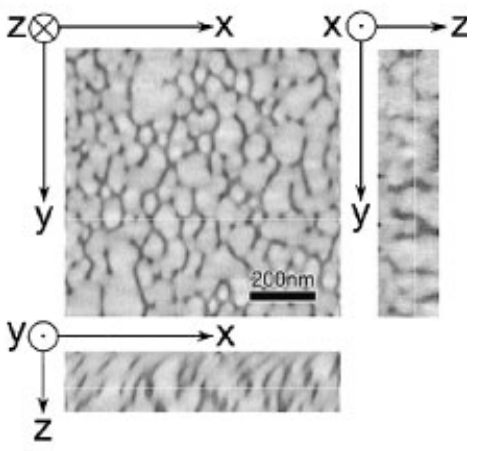

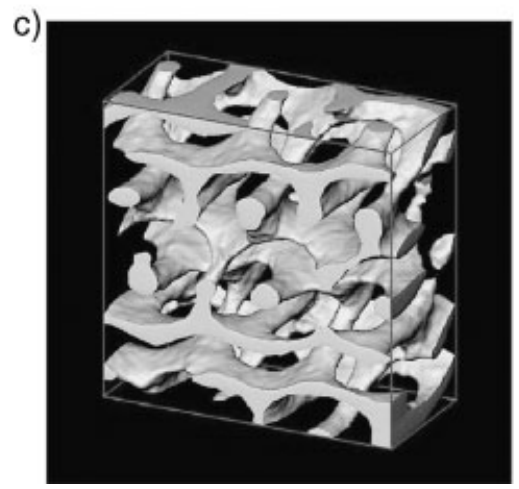

d)

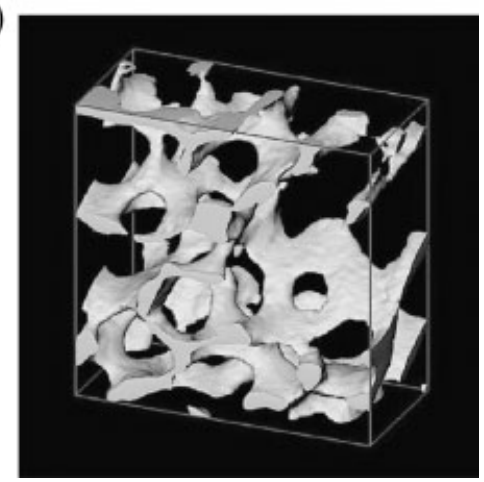

Figure 2. Two-dimensional planar $(x, y)$ and cross-sectional $(x, z ; y, z)$ slices of the a) ordered and b) disordered bicontinuous morphologies generated from 3D TEMT images. The positions from which the slices have been extracted are identified in the images. Included in c) and d) are the corresponding 3D solid renditions of the I microdomains within a transparent $\mathrm{S} / \mathrm{hS}$ matrix. 
image acquisition are detected by the magnitude of the average root mean square (rms) alignment error $(\Delta)$, which relates the observed $(x, y, \theta)$ position of each Au reference marker to its expected position known from the $0^{\circ}$ tilt image. Calculated values of $\Delta, 0.13$ and $0.18 \mathrm{~nm}$ for the ordered and disordered morphologies, respectively, are acceptably low to permit further analysis of the TEMT images.

Although the bulk composition of the present blend is known (29-30 vol.-\% I), the composition of each coexisting phase is not. Since TEMT analysis over the present angular range ${ }^{[20 a]}$ can assess composition within $3 \%$ of the known composition, it is used to analyze the morphologies in Figure 2. Measured compositions in Figure 2a,c and 2b,d are 21 and 15 vol.- $\%$ I, respectively. The former corresponds to 51 vol.- $\% \mathrm{hS}$, whereas the composition of the disordered morphology spatially varies. These compositions are generally lower in the minority component than those reported for ordered bicontinuous morphologies in linear block copolymers and their blends. The amount of each phase present in the blend cannot be determined because the disordered morphology is spatially heterogeneous, displaying evidence of $\mathrm{hS}$ enrichment along the ordered/disordered interface. Fourier analysis of the ordered morphology yields the crystallographic identity and characteristic size of the unit cell. Peak positions located at $1: \sqrt{6}: \sqrt{8}$ in the 3D Fourier transform of the reconstructed solid used to generate Figure $2 \mathrm{c}$ corroborate that the morphology is $\mathrm{G}$ with a mean unit cell size of 122 $\mathrm{nm}$. Conversely, Fourier analysis of the disordered bicontinuous morphology yields a diffuse ring with a much lower interdomain distance $(L)$ of $\approx 67 \mathrm{~nm}$. Skeletonization of the $3 \mathrm{D}$ reconstructions in Figure $2 \mathrm{c}$ and $2 \mathrm{~d}$ further reveals that the channel coordination is primarily (89\%) 3 in both morphologies, thereby confirming that the ordered morphology is G. This observation indicates that the $\mathrm{G}$ morphology can develop at relatively high $\chi N$, which is consistent with experimental observations of chemically modified copolymers ${ }^{[29]}$ and recent SCFT developments. ${ }^{[30]}$ A second outcome is that the Euler number (Eu), given by $2-2 g$ (where $g$ denotes genus), is measured as $-14 \pm 0.4$ for the $\mathrm{G}$ morphology (which is comparable to that of the $\mathrm{G}$ morphology in a neat SIS copolymer, $-14 \pm 0.3$ ) and $-3.4 \pm 0.2$ for the disordered bicontinuous morphology. For reference, the value of $\mathrm{Eu}$ for the $\mathrm{G}$ morphology derived $^{[20 b]}$ earlier for a composition-matched Schoen $G$ minimal surface is $-16 \pm 0.2$.

The local shape of any curved surface can be described by two principal curvatures $\kappa_{1}$ and $\kappa_{2}$, which together yield the mean curvature $H$, given by $\left(\kappa_{1}+\kappa_{2}\right) / 2$, and the Gaussian curvature $K$, defined here as $\kappa_{1} \kappa_{2}$. The area-averaged mean curvature $(\langle\mathrm{H}\rangle)$ governs global morphological development, whereas the corresponding standard deviation $\left(\sigma_{\mathrm{H}}\right)$ relates to interfacial packing frustration and the thermodynamic stability of bicontinuous morphologies. ${ }^{[4]}$ The probability density of $H$, designated $P(H)$, is derived from

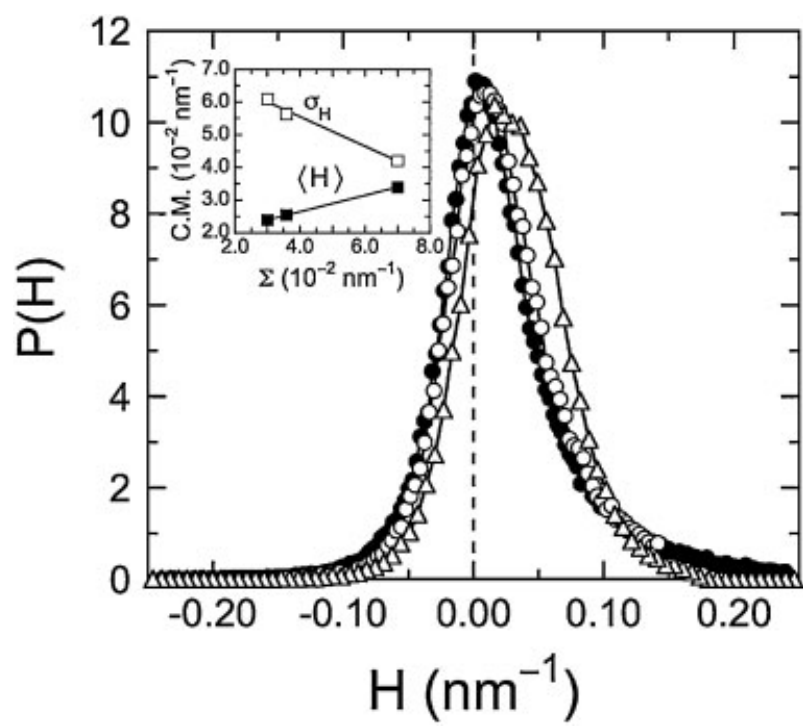

Figure 3. Probability density of the mean curvature $(H)$ generated from TEMT images of the ordered $(O)$ and disordered (O) bicontinuous morphologies in Figure 1 and 2. Included are data for the G morphology in a neat SIS copolymer $(\triangle) \cdot{ }^{[20]}$ The solid lines connect the data, and the dashed line identifies $H=0$. The inset shows the relationship between interfacial area/volume $(\Sigma)$ and two curvature metrics (C.M.): $\sigma_{\mathrm{H}}(\square)$ and $\langle\mathrm{H}\rangle(\boldsymbol{\square})$. The solid lines are linear regressions.

a joint interfacial curvature probability distribution determined by measuring $H$ and $K$ at random points along the interface. The $P(H)$ distributions generated for the ordered $(G)$ and disordered bicontinuous morphologies evident in Figure 1 and 2 are presented in Figure 3 and appear strikingly similar: both are nearly Gaussian in shape but not centered at $H=0$. Included for comparison is $P(H)$ reported $^{[20 \mathrm{a}]}$ for a neat SIS triblock copolymer that exhibits the $\mathrm{G}$ morphology. It resembles the probability densities obtained from the SI/hS blend, but is shifted further from $H=0$. The $P(H)$ data in Figure 3 indicate that the magnitude of $\langle\mathrm{H}\rangle$ increases with increasing nanostructural order. Another quantity that reflects the extent of molecular organization is the interfacial area/volume $(\Sigma)$. Although a limited number of data points are available, the inset included in Figure 3 shows that i) an increase in $\Sigma$ promotes an increase in $\langle\mathrm{H}\rangle$ and a decrease in $\sigma_{\mathrm{H}}$, and ii) the $\mathrm{G}$ morphology in the $\mathrm{SI} / \mathrm{hS}$ blend possesses a lower $\Sigma$ (and is presumably more defective) than the one in the SIS copolymer.

While direct visualization and quantitative analysis of TEMT images can provide real-space insight into the structural characteristics of complex nanometer-scale morphologies, the spatial distribution of the constituent species within the present SI/hS blend cannot be extracted from TEMT images. The TEMT images can, however, be complemented by results from molecular-field calculations, examples of which are displayed in Figure 4. These 


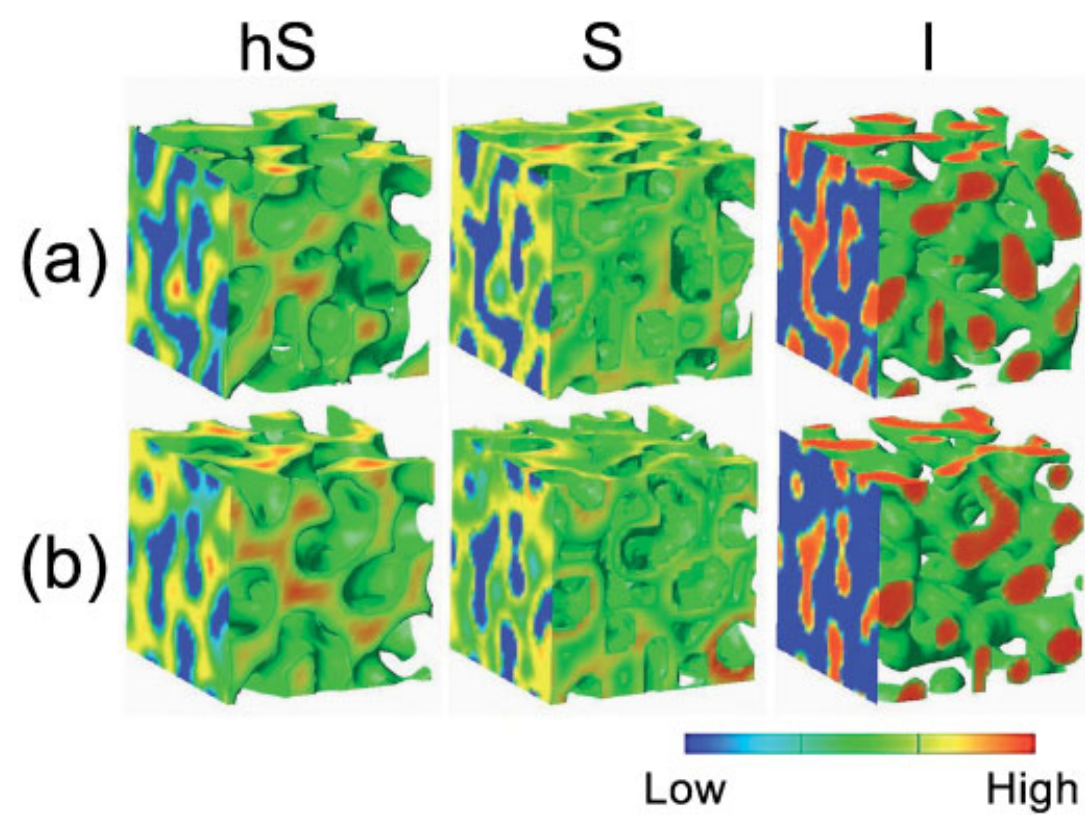

Figure 4. MesoDyn simulations of SI/hS blends that differ in composition (vol.-\% hS): a) 30 and b) 34 . The color scale denotes the concentration of hS (left), S (middle), or I (right). These simulations were performed with a single S-I interaction parameter and did not differentiate between $\mathrm{S}$ units along the copolymer or homopolymer chains. The time increment $(\Delta t)$ depends on the diffusivity $(D)$ and grid size $(\Delta x)$ by $\Delta t=0.009 \Delta x^{2} / D$

calculations have been performed for SI/hS blends with two different $\mathrm{hS}$ volume fractions, 0.30 (Figure $4 \mathrm{a}$ ) and 0.34 (Figure $4 \mathrm{~b}$ ), to ascertain the influence of composition on predicted phase behavior by using the MesoDyn software package, which considers dynamically evolving molecular fields and which, unlike equilibrium SCFT, can elucidate highly defective morphologies and mechanistic pathways. ${ }^{[31]}$ The experimental composition of the blend is calculated to be $\approx 31$ vol.- $\%$ hS. The simulations illustrating the I distribution can be compared directly with the TEMT images, since the stain-induced contrast in TEM delineates the styrene and diene moieties. It is important to recognize that the I microdomains are irregularly shaped, with some possessing a near-circular cross-section and others appearing more layer-like. This characteristic is consistent with the 2D and 3D TEMT images provided in Figure $2 b$ and $2 d$, respectively. Close examination of the $\mathrm{hS}$ and $\mathrm{S}$ distributions included in Figure 4 reveals that a large fraction of the imbibed hS tends to locate near the center of the $\mathrm{S} / \mathrm{hS}$ microdomains. At 34 vol.- $\% \mathrm{hS}$, evidence of macrophase separation, which lowers the concentration of $\mathrm{hS}$ in one phase while increasing it in the other, is observed in the simulations. This result is consistent with the experimental morphologies displayed in Figure 1 and 2. As expected, the $\mathrm{S}$ blocks reside primarily at the interface separating the I-rich channels from the hS-rich matrix.

Fourier analysis of the MesoDyn simulations shows that $L$ gradually increases from 74 to $94 \mathrm{~nm}$ with increasing calculation time at 30 vol.- $\% \mathrm{hS}$, and quickly increases from
77 to $155 \mathrm{~nm}$ at 34 vol.- $\% \mathrm{hS}$. At the high hS concentration, macrophase separation occurs and the range over which $L$ swells during coarsening spans the measured unit cell sizes of both morphologies in Figure 1 and 2. An ordered bicontinuous morphology does not, however, develop even after longer $(12.5 \times)$ calculation times. While long-range order may eventually evolve, this observation may alternatively indicate that the free energy difference between the ordered and disordered bicontinuous morphologies is not large or that slow phase-separation kinetics permit dynamically-evolving intermediate structures or fluctuations near the order-disorder transition ${ }^{[32]}$ to be frozen-in. Irrespective of the degree to which phase equilibrium is attained, the combination of quantitative TEMT analysis ${ }^{[20,21,33,34]}$ and molecular-field modeling ${ }^{[31]}$ provides a powerful set of tools by which to examine the structural attributes and molecular arrangement of complex structural motifs.

\section{Conclusion}

Coexistence of ordered and disordered bicontinuous morphologies in block copolymer/homopolymer blends supports the SCFT predictions of Matsen, ${ }^{[25]}$ and further implies that the degree of order in such a system may be physically adjusted. Composition-tunable bicontinuous order augments existing paradigms established for morphologies and nanostructural dimensions, and may ultimately provide a facile means by which to achieve desirable 
transport or mechanical properties. The analyses reported here establish the structural similarities and differences between coexisting ordered and aperiodic bicontinuous morphologies in a block copolymer/homopolymer blend. This unique blend further elucidates the complex phase behavior of block copolymer systems and likewise constitutes a good physical model of coexisting bicontinuous phases encountered in biological systems (e.g., polymorphs observed in plant cytomembranes ${ }^{[35]}$ ).

Acknowledgements: This study was supported by the Japanese Ministry of Economy, Trade and Industry (HJ), the Japan Society for the Promotion of Science $(\mathrm{HH})$, the National Institutes of Health and the W. M. Keck Foundation (DAA), the U.S. Department of Energy and, in part, the Research Council of Norway under the NANOMAT Program (RJS). Supercomputer time for the MesoDyn calculations was provided by Stichting Nationale Computer Faciliteiten (NCF). We thank Dr. D. J. Frankowski for technical assistance.

[1] I. W. Hamley, "The Physics of Block Copolymers", Oxford University Press, New York 1998.

[2] C. Park, J. Yoon, E. L. Thomas, Polymer 2003, 44, 6725.

[3] I. W. Hamley, Angew. Chem. Int. Ed. 2003, 42, 1692.

[4] M. W. Matsen, F. S. Bates, J. Chem. Phys. 1997, 106, 2436.

[5] N. Hadjichristidis, S. Pispas, G. A. Floudas, "Block Copolymers: Synthetic Strategies, Physical Properties, and Applications", Wiley-Interscience, New York 2003.

[6] M. W. Matsen, F. S. Bates, J. Polym. Sci., Part B: Polym. Phys. 1997, 35, 945.

[7] J. T. Chen, E. L. Thomas, C. K. Ober, G. P. Mao, Science 1996, 273, 343

[8] J. P. A. Fairclough, S. M. Mai, M. W. Matsen, W. Bras, L. Messe, S. C. Turner, A. J. Gleeson, C. Booth, I. W. Hamley, A. J. Ryan, J. Chem. Phys. 2001, 114, 5425.

[9] J. Raez, I. Manners, M. A. Winnik, J. Am. Chem. Soc. 2002 $124,10381$.

[10] R. J. Spontak, N. P. Patel, "Developments in Block Copolymer Science and Technology", I. W. Hamley, Ed., Wiley, New York 2004, pp. 159-212.

[11] L. Zhu, P. Huang, S. Z. D. Cheng, Q. Ge, R. P. Quirk, E. L. Thomas, B. Lotz, J. C. Wittmann, B. S. Hsiao, F. J. Yeh, L. Z. Liu, Phys. Rev. Lett. 2001, 86, 6030.

[12] A. C. Finnefrock, R. Ulrich, A. Du Chesne, C. C. Honeker, K. Schumacher, K. K. Unger, S. M. Gruner, U. Wiesner, Angew. Chem. Int. Ed. 2001, 40, 1208.

[13] M. F. Schulz, F. S. Bates, K. Almdal, K. Mortensen, Phys. Rev. Lett. 1994, 73, 86
[14] D. A. Hajduk, P. E. Harper, S. M. Gruner, C. C. Honeker, G. Kim, E. L. Thomas, L. J. Fetters, Macromolecules 1994, 27, 4063.

[15] J. H. Laurer, D. A. Hajduk, J. C. Fung, J. W. Sedat, S. D. Smith, S. M. Gruner, D. A. Agard, R. J. Spontak, Macromolecules 1997, 30, 3938.

[16] Y. Tselikas, N. Hadjichristidis, R. L. Lescanec, C. C. Honeker, M. Wohlgemuth, E. L. Thomas, Macromolecules 1996, 29, 3390.

[17] T. P. Lodge, B. Pudil, K. J. Hanley, Macromolecules 2002 , $35,4707$.

[18] [18a] K. I. Winey, E. L. Thomas, L. J. Fetters, Macromolecules 1992, 25, 422; [18b] K. I. Winey, E. L. Thomas, L. J. Fetters, Macromolecules 1992, 25, 2645.

[19] R. J. Spontak, J. C. Fung, M. B. Braunfeld, J. W. Sedat, D. A. Agard, L. Kane, S. D. Smith, M. M. Satkowski, A. Ashraf, D. A. Hajduk, S. M. Gruner, Macromolecules 1996, 29, 4494.

[20] [20a] H. Jinnai, Y. Nishikawa, R. J. Spontak, S. D. Smith, D. A. Agard, T. Hashimoto, Phys. Rev. Lett. 2000, 84, 518; [20b] H. Jinnai, T. Kajihara, H. Watashiba, Y. Nishikawa, R. J. Spontak, Phys. Rev. E 2001, 64, 10803.

[21] H. Jinnai, Y. Nishikawa, M. Ito, S. D. Smith, D. A. Agard, R. J. Spontak, Adv. Mater. 2002, 14, 1615.

[22] P. Falus, H. Xiang, M. A. Borthwick, T. P. Russell, S. G. J. Mochrie, Phys. Rev. Lett. 2004, 93, 145701.

[23] F. S. Bates, W. W. Maurer, P. M. Lipic, M. A. Hillmyer, K. Almdal, K. Mortensen, G. H. Fredrickson, T. P. Lodge, Phys. Rev. Lett. 1997, 79, 849.

[24] S. Jain, F. S. Bates, Science 2003, 300, 460.

[25] [25a] M. W. Matsen, Macromolecules 1995, 28, 5765; [25b] M. W. Matsen, Macromolecules 2003, 36, 9647.

[26] A. J. Koster, M. B. Braunfeld, J. C. Fung, C. K. Abbey, K. F. Han, W. Liu, H. Chen, J. W. Sedat, D. A. Agard, EMSA Bull. 1993, 23, 176.

[27] T. P. Lodge, C. Pan, X. Jin, Z. Liu, J. Zhao, W. W. Maurer, F. S. Bates, J. Polym. Sci., Part B: Polym. Phys. 1995, 33, 2289.

[28] S. W. Sides, G. H. Fredrickson, J. Chem. Phys. 2004, 121, 4974.

[29] D. A. Davidock, M. A. Hillmyer, T. P. Lodge, Macromolecules 2004, 37, 397.

[30] E. W. Cochran, C. J. Garcia-Cervera, G. H. Fredrickson, Macromolecules 2006, 39, 2449.

[31] B. A. C. van Vlimmeren, N. M. Maurits, A. V. Zvelindovsky, G. J. A. Sevink, J. G. E. M. Fraaije, Macromolecules 1999, $32,646$.

[32] J. Wang, Z.-G. Wang, Y. Yang, Macromolecules 2005, 38, 1979.

[33] H. Jinnai, Y. Nishikawa, T. Ikehara, T. Nishi, Adv. Polym. Sci. 2004, 170, 115

[34] V. Abetz, R. J. Spontak, Y. Talmon, "Macromolecular Engineering: From Precise Macromolecular Synthesis to Macroscopic Materials Properties and Applications", K. Matyjaszewski, Y. Gnanou, L. Leibler, Eds., Wiley-VCH, Weinheim 2006, Vol. 3, Ch. 9.

[35] S. Hyde, S. Andersson, K. Larsson, Z. Blum, T. Landh, S. Lidin, B. W. Ninham, "The Language of Shape", Elsevier Science, Amsterdam 1997, Ch. 7. 